Proc. of 12th International Workshop on Positron and Positronium Chemistry, August 28-September 1, 2017, Lublin, Poland

\title{
Preliminary Studies of J-PET Detector Spatial Resolution
}

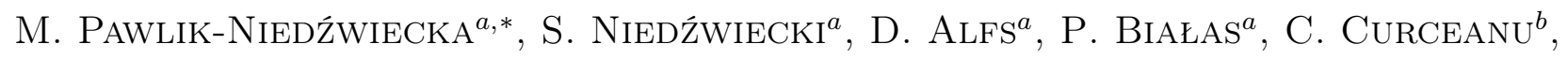

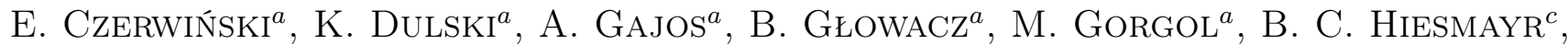

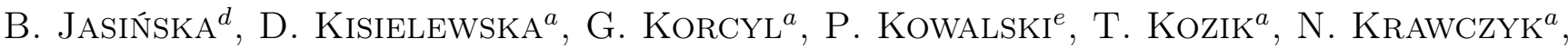
W. Krzemień ${ }^{f}$, E. Kubicz ${ }^{a}$, M. Mohammed ${ }^{a, g}$, M. PAŁKA $^{a}$, L. RACZYŃski $^{e}$, J. RAJ $^{a}$, Z. Rudy ${ }^{a}$, Shivani $^{a}$, M. Silarski $^{a}$, M. Skurzok ${ }^{a}$, N. G. Sharma ${ }^{a}$, S. Sharma $^{a}$, R.Y. Shopa ${ }^{e}$, A. Strzelecki ${ }^{a}$, A. Wieczorek ${ }^{a}$, W. Wiślicki ${ }^{f}$, B. ZGARdzińskA $^{d}$, M. ZIELIŃSKI ${ }^{a}$ AND P. MOSKAL ${ }^{a}$

${ }^{a}$ Institute of Physics, Jagiellonian University, Łojasiewicza 11, 30-348 Kraków, Poland

${ }^{b}$ INFN, Laboratori Nazionali di Frascati, 00044 Frascati, Italy

${ }^{c}$ Faculty of Physics, University of Vienna, 1090 Vienna, Austria

${ }^{d}$ Institute of Physics, Maria Curie-Skłodowska University, 20-031 Lublin, Poland

${ }^{e}$ Laboratory for Information Technologies, National Centre for Nuclear Research, 05-400 Otwock-Świerk, Poland

${ }^{f}$ High Energy Physics Division, National Centre for Nuclear Research, 05-400 Otwock-Świerk, Poland

${ }^{g}$ Department of Physics, College of Education for Pure Sciences, University of Mosul, Mosul, Iraq

The J-PET detector, based on long plastic scintillator strips, was recently constructed at the Jagiellonian University. It consists of 192 modules axially arranged into three layers, read out from both sides by digital constant-threshold front-end electronics. This work presents preliminary results of measurements of the spatial resolution of the J-PET tomograph performed with ${ }^{22} \mathrm{Na}$ source placed at selected position inside the detector chamber.

DOI: 10.12693/APhysPolA.132.1645

PACS/topics: 29.40.Mc, 87.57.uk, 87.10.Rt, 34.50.-S

\section{Introduction}

The Jagiellonian Positron Emission Tomograph (JPET) is under continuous development at the Jagiellonian University [1-9]. This novel PET system is based on three layers of axially arranged plastic scintillator strips, which form a cylindrical chamber [9]. Signals from scintillators are read-out by vacuum tube photomultipliers placed at two opposite ends of each strip and are probed by dedicated multi-threshold front-end electronics $[5,7]$.

Novelty of this prototype is based on the utilization of organic scintillators as a radiation detectors instead of expensive crystals [1,4]. All presently available PET scanners are based on crystal scintillators providing that only around $20 \mathrm{~cm}$ of patient body can be examined in one position of bed $[10,11]$. To make a whole-body scan it is necessary to make from 8 to 11 examinations [Karp]. The J-PET prototype is developed in order to build a whole-body scanner which will allow to decrease the measurement time and, in consequence, the radiation dose received by a patient. Organic scintillators utilized in J-PET can be extended to as much as $200 \mathrm{~cm} \mathrm{[1,2].}$

\footnotetext{
* corresponding author; e-mail: monika.pawlik@doctoral.uj.edu.pl
}

The cost-effective J-PET prototype is optimized for detection of photons from electron-positron annihilations. Place and time of $\gamma$ particle interaction with scintillator material is determined by time difference calculations between two ends of the same scintillator strip.

Determination of the J-PET performance characteristics is necessary in order to check if it can be used in clinical application at hospitals [13]. NEMA standards allow to define a guaranteed quality of measurement results and to compare the performance characteristics of systems available from various manufacturers. NEMA defines scanner parameters such as spatial resolution, scatter fraction, count losses, sensitivity, image quality, accuracy of attenuation and scatter corrections and provides description of a set of measurements needed for tests according to this standard. Results of first simulations of the NEMA parameters with the J-PET detector were presented in articles [14-16].

\section{Determination of the J-PET spatial resolution}

Spatial resolution of a scanner characterizes the possibility of the imaging system to distinguish two points from each other after reconstruction of the image and specifies the highest achievable performance characteristic.

For spatial resolution studies a single point source should be measured in air and reconstructed with no smoothing or apodization. The main aim of this kind of 
measurements is to obtain an image of the point source referred to as point spread function (PSF). PSF is used as a characteristics of the spatial resolution of the scanner.

\subsection{Measurements}

A ${ }^{22} \mathrm{Na}$ point source was placed at six positions inside the J-PET prototype according to the NEMA-NU-2-2012 standard: in the $y$ direction at $1 \mathrm{~cm}, 10 \mathrm{~cm}$ and $20 \mathrm{~cm}$ for the center of the axial field-of-view (FOV) and for three-eights of the axial FOV from the center (Fig. 1 and Fig. 2). Therefore, source was placed at following positions: $(0,1,0),(0,10,0),(0,20,0),(0,1,-18.75)$, $(0,10,-18.75)$ and $(0,20,-18.75)$. Position $(0,0,0)$ is the geometrical center of the J-PET prototype in $x, y$ and $z$ directions. The width and thickness of active part of source were equal to $3 \mathrm{~mm}$ and $1 \mathrm{~mm}$, respectively.

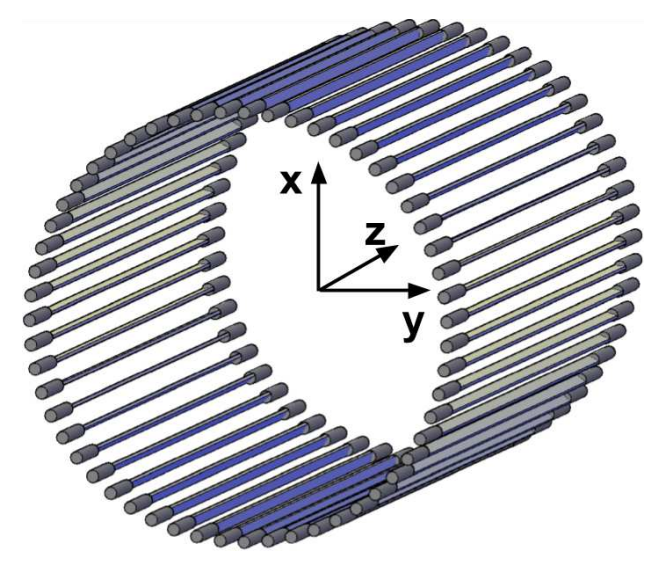

Fig. 1. J-PET reference frame used during offline data analysis and reconstruction.

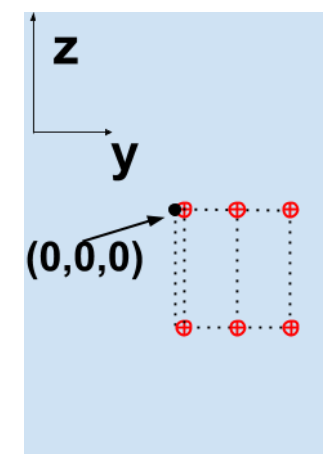

Fig. 2. Position marked on styrofoam panel according to NEMA standard. Distance between red dots in the $\mathrm{z}$ direction is equal to $18.75 \mathrm{~cm}$ while distance between first and second and third dot in $y$ direction is equal to $9 \mathrm{~cm}$ and $19 \mathrm{~cm}$, respectively.

A dedicated styrofoam panel was prepared for measurements and was hanged parallel to the long axis ( $\mathrm{z}$ axis) of the J-PET prototype and centred in each direction. Styrofoam was chosen because of its low density and small probability for scattering of gamma quanta on the panel.

\subsection{Data selection criteria}

Collected data were analysed with a dedicated analysis framework created in $\mathrm{C}++$ language [17]. For each event registered time values were corrected with time calibration constants and effective light velocity in scintillators. In this preliminary analysis the hit-time and hit-position was determined based only on the measurements at one (the lowest) threshold. More advanced procedures for the application of all thresholds are under evaluation [18-20].

The field of view of the J-PET prototype in $x$ and $y$ directions was limited to circle with diameter equal to $50 \mathrm{~cm}$. Consequently, only those events were accepted for the reconstruction for which the distance between the LOR (line-of-response, line connecting two opposite hits) and the centre of the tomograph was smaller than $25 \mathrm{~cm}$ (Fig. 3).

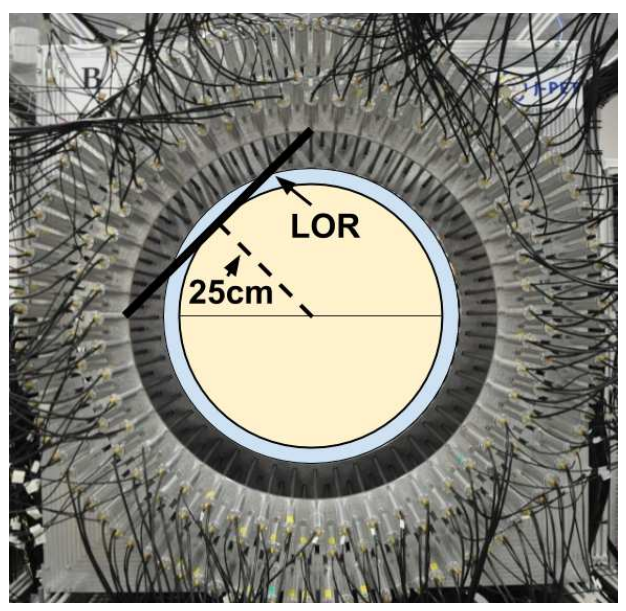

Fig. 3. A photo of the J-PET tomograph. Superimposed "yellow" circle indicate a FOV accepted during data analysis.

Data were also selected using the criterion based on the reconstructed energy loss. The energy loss was estimated by the sum of time-over-thresholds (TOT) values measured at four thresholds by two photomultipliers connected to the scintillator. Fig. 4 shows the histogram of the sum of TOT values from four threshold from both sides of each scintillator in the J-PET prototype.

Lower limit on TOT corresponds to about $200 \mathrm{keV}$ energy deposition. Applying this criterion reduces contribution of counts that originate from secondary photon scatterings in the detector material to the acceptable level of $1 \%$. This is caused by the energy limit of 184 $\mathrm{keV}$, which can be deposited during the second scattering [15]. The upper limitation of the TOT value reduces contribution from the $1270 \mathrm{keV}$ photon originating from the ${ }^{22} \mathrm{Ne}$ de-excitation.

Additionally, the minimum angular distance between two strips which registered $\gamma$ quanta was set to 20 degrees. This condition allowed for complete reduction of 


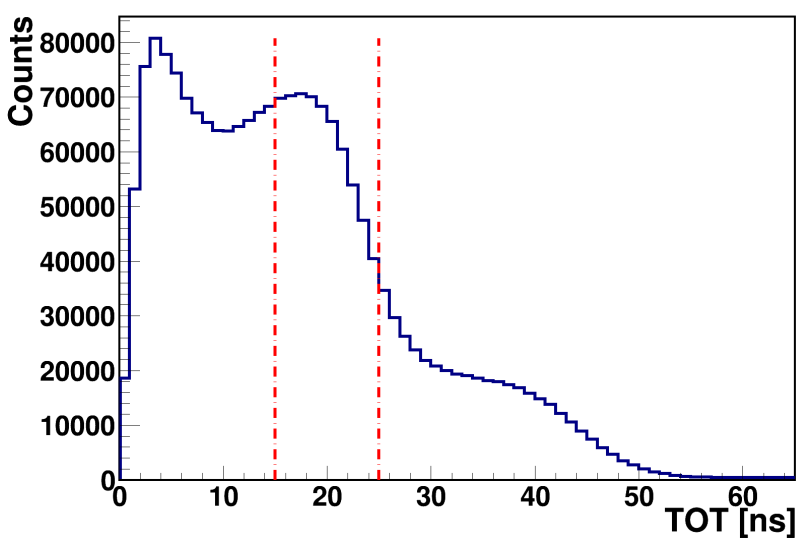

Fig. 4. Exemplary histogram of the sum of TOT for all scintillators for source placed in position $(0,1,0)$. Applied cuts are marked with dotted line.

scatterings between strips close to each other. Moreover, $\mathrm{z}$ coordinate of place of interaction of $\gamma$ quantum with strip and $\mathrm{z}$ coordinate of calculated place of annihilation was limited to $\pm 23 \mathrm{~cm}$ due to J-PET geometry. Events with larger value of $\mathrm{z}$ coordinate were rejected. This limitation allowed for rejection of gamma quanta scattered on mounting plates.

\section{Results}

Exemplary results of the distributions of place of annihilation for source placed in position $(0,1,0)$ before and after data selection are shown in Fig. 5 and Fig. 6. Applied cuts allowed for partial noise rejection.

Time and position of each gamma quantum reaction with scintillator material was used in image reconstruction performed by dedicated 3D MLEM algorithm [21].

A PSF function, for the highest bin from reconstructed image of source, was drawn in all three directions. FWHM (full width at half maximum) of each profile is reported as the J-PET spatial resolution. Preliminary result for source placed in $(0,1,0)$ one can find in Fig. 7.

Spatial resolution along $x$ and $y$ axis is of the order of pixel size $(1 \mathrm{~mm})$ set in MLEM reconstruction algorithm and is equal to about $2-3 \mathrm{~mm}$ due to the source size. Resolution along $\mathrm{z}$ axis, in the worst case, is of the order of $25 \mathrm{~mm}$ (see Table I) and can be improved by using more than one threshold for estimation of TOF (time of flight) and optimization of TOT cut values.

Transaxial and axial resolution of currently available $\mathrm{PET} / \mathrm{CT}$ scanners is of the order of $4-5 \mathrm{~mm}$ for positions of source close to the center of FOV (see Table II). Preliminary result of J-PET spatial resolution along $x$ and $y$ direction is comparable with parameters achieved by commercial PET scanners. In future, the spatial resolution along $\mathrm{z}$ axis can be improved by an an application of the array of WLS (wavelenght-shifting) strips placed perpendicularly to scintillation modules and by utilization of SiMP (silicon photomultiplier) readout $[4,8]$.
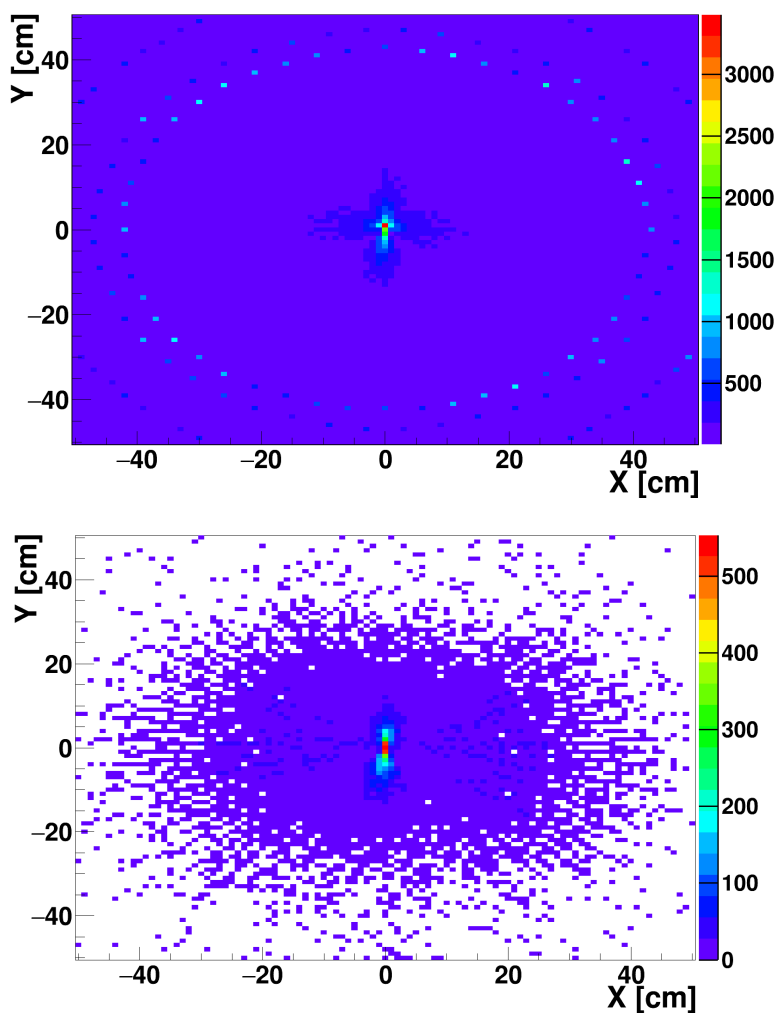

Fig. 5. Place of annihilation in $x$ and $y$ direction before (top) and after (bottom) a selection criteria were applied.
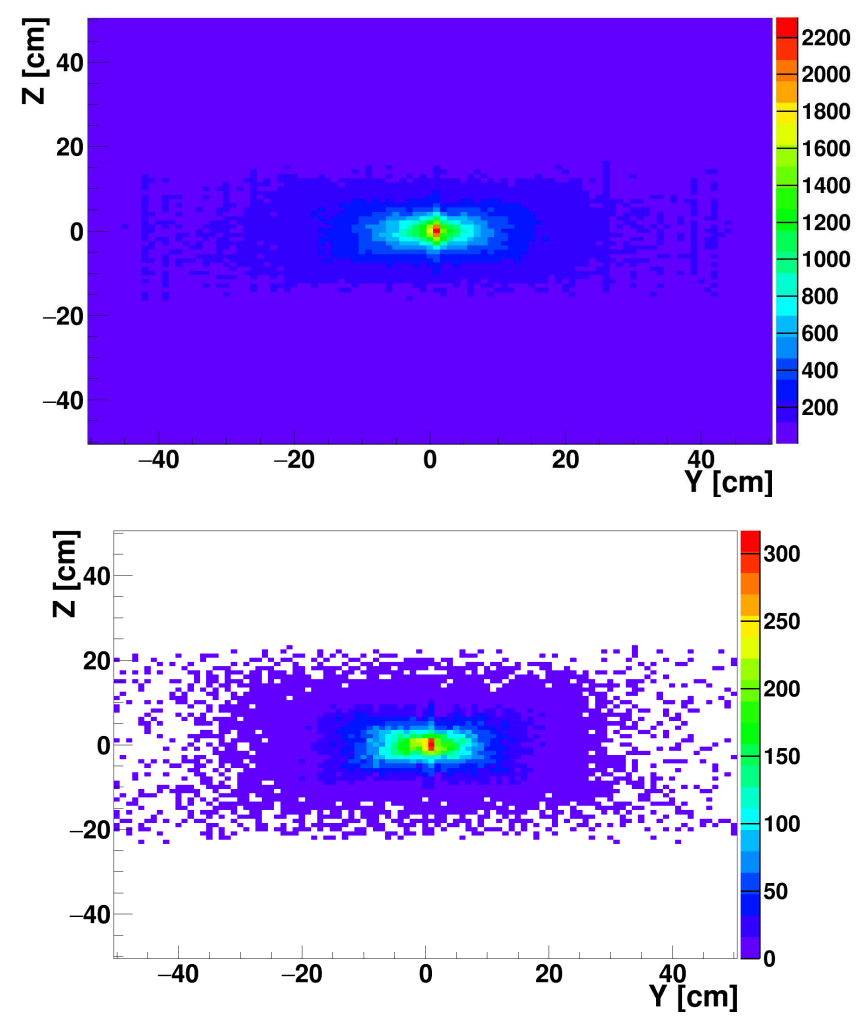

Fig. 6. Place of annihilation in $y$ and $\mathrm{z}$ direction before (top) and after (bottom) application of selection criteria. 

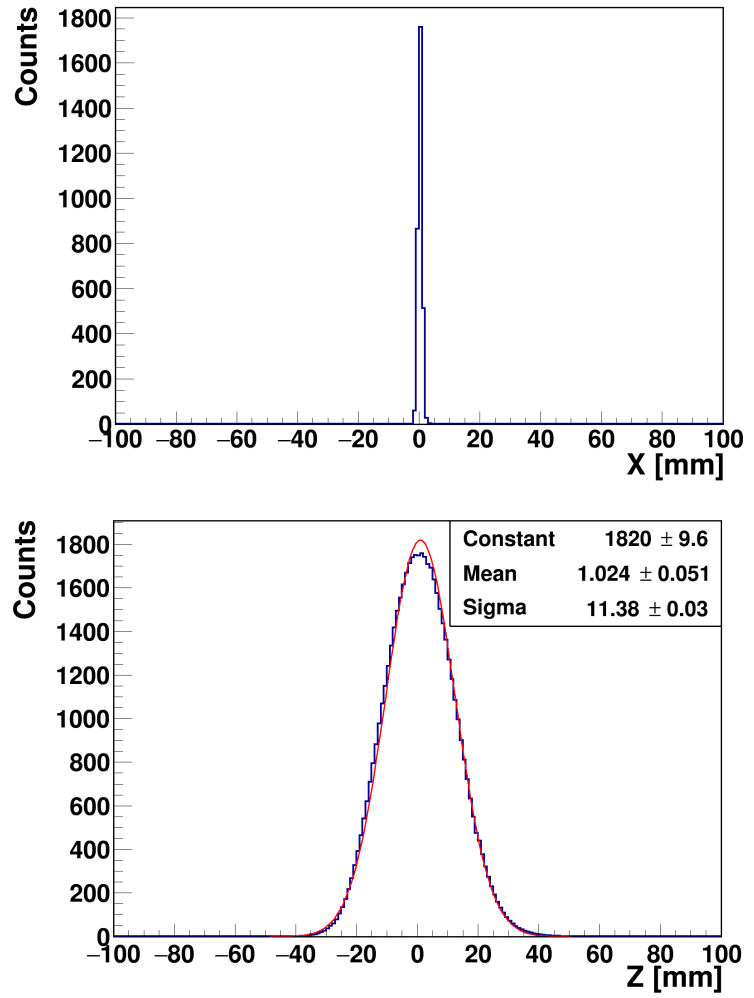

Fig. 7. PSF function in $x$ and $\mathrm{z}$ direction.

TABLE I

Results of sigma $z$ along $z$ direction for source in six positions.

\begin{tabular}{|c|c|}
\hline Source position & Sigma $\mathrm{z}[\mathrm{mm}]$ \\
\hline$(0,1,0)$ & $11.38 \pm 0.03$ \\
\hline$(0,10,0)$ & $9.84 \pm 0.07$ \\
\hline$(0,20,0)$ & $7.77 \pm 0.08$ \\
\hline$(0,1,-18.75)$ & $9.99 \pm 0.05$ \\
\hline$(0,10,-18.75)$ & $7.77 \pm 0.09$ \\
\hline$(0,20,-18.75)$ & $7.35 \pm 0.08$ \\
\hline
\end{tabular}

TABLE II Tranaxial and axial resolution at $1 \mathrm{~cm}$ and $10 \mathrm{~cm}$ for different models of PET/CT scanners [10].

\begin{tabular}{|c|c|c|c|}
\hline PET/CT Model & $\begin{array}{c}\text { Philips } \\
\text { Ingenuity TF }\end{array}$ & $\begin{array}{c}\text { GE } \\
\text { Discovery } \\
\mathbf{7 1 0}\end{array}$ & $\begin{array}{c}\text { Siemens } \\
\text { Biograph } \\
\text { mCT Flow }\end{array}$ \\
\hline $\begin{array}{c}\text { Transaxial } \\
\text { resolution @ 1 } \\
\text { cm [mm] }\end{array}$ & 4,7 & 4,9 & 4,4 \\
\hline $\begin{array}{c}\text { Transaxial } \\
\text { resolution @ 10 } \\
\text { cm [mm] }\end{array}$ & 5,2 & 5,5 & 4,9 \\
\hline $\begin{array}{c}\text { Axial resolution } \\
\text { @ 1 cm [mm] }\end{array}$ & 4,7 & 5,6 & 4,5 \\
\hline $\begin{array}{c}\text { Axial resolution } \\
\text { @ 10 cm [mm] }\end{array}$ & 5,2 & 6,3 & 5,9 \\
\hline
\end{tabular}

\section{Acknowledgments}

The authors acknowledge the technical support by A. Heczko, W. Migdał, and the financial support from the Polish National Center for Development and Research through grant INNOTECHK1/IN1/64/159174/NCBR/12, Marian Smoluchowski Kraków Research Consortium "Matter-Energy-Future", the EU and MSHE Grant no. POIG.02.03.00-161 00013/09, National Science Center Poland through grant No. 2016/21/B/ST2/01222 and by the Ministry for Science and Higher Education through grants No. 7150/E338/M/2017 and 7150/E-338/SPUB/2017/1. B. C. Hiesmayr acknowledges gratefully the Austrian Science Fund FWF-P26783.

\section{References}

[1] P. Moskal et al., Bio-Algorithms and Med-Systems 7 , 73 (2011).

[2] P. Moskal et al., Nucl. Instr. and Meth. A 764, 317 (2014).

[3] P. Moskal et al., Nucl. Instr. and Meth. A 775, 54 (2015).

[4] P. Moskal et al., Phys. Med. Biol. 61, 2025 (2016).

[5] G. Korcyl et al., Acta Phys. Polon. B 47, 491 (2016).

[6] P. Moskal et al., Acta Phys. Polon. B47, 509 (2016).

[7] M. Pałka et al., JINST 12, P08001 (2017).

[8] J. Smyrski et al., Nucl. Instr. and Meth. A 851, 39 (2017).

[9] S. Niedźwiecki et al., Acta Phys. Polon. B 48, 1567 (2017).

[10] P.J. Slomka et al., Semin. Nucl. Med. 46, 5 (2016).

[11] S. Vandenberghe et al., EJNMMI Phys. 3, 3 (2016).

[12] J.S. Karp et al., J. Nucl. Med. 49, 462 (2008).

[13] National Electrical Manufacturers Association, Norm NEMA-NU-2 Performance Measurements of Positron Emission Tomographs (PETs), 2012.

[14] P. Kowalski et al., Acta Phys. Pol. A 127, 1505 (2015).

[15] P. Kowalski et al., Acta Phys. Polon. B 47, 549 (2016).

[16] R. Shopa et al., Acta Phys. Polon. B 48, 1757 (2017).

[17] W. Krzemień et al., Acta Phys. Polon. B 47, 561 (2016).

[18] L. Raczyński et al., Nucl. Instr. and Meth. A 764, 186 (2014).

[19] L. Raczyński et al., Nucl. Instr. and Meth. A $\mathbf{7 8 6}$, 105 (2015).

[20] L. Raczyński et al., Phys. Med. Biol. 62, 5076 (2017).

[21] A. Strzelecki, Ph.D. Thesis, Jagiellonian University, KrakĂłw 2016 